\title{
Display elements and gaps: a comparison of flat panel display characteristics
}

\author{
G.P.J. Spenkelink and J. Besuijen
}

\begin{abstract}
The relation between typical flat panel display characteristics and display quality was studied. Subjective preferences were obtained with respect to simulated black-on-white flat panel displays. The displays differed in the sort of separation between the display elements and the shape of these elements. Further, the height/width ratio of the front was studied in relation with a fixed font matrix. The preferences were obtained through a paired comparison of all possible pairs of simulated displays.

The observers appeared to be sensitive and to respond to differences in the stimuli. It is not clear, however, if they responded to the type of gap and shape of display elements or to the luminance modulation, which co-varied with the two experimental factors. It can be concluded however that the integrity of the character area is important to the observers. Further, the usefulness of interactively designing spatial display characteristics and the font is discussed.
\end{abstract}

Keywords: display quality, flat panel displays, display standards, design rules, simulation

There is currently a great deal of interest in flat panel displays (FPDs) and much effort is being put into further development of FPD technologies. FPDs have great potential for a number of reasons (eg. portability, power requirements), but from the ergonomist's point of view there are also several problems that are more or less uniquely associated with these display technologies. To be able to deal with the human factor in the development and design of FPDs as part of the human-machine interface, there is a need to investigate the ergonomic aspects of such displays.

Ultimately, it is the compatibility of a display's visual properties with the properties of the human visual system and with the human information processing characteristics that determines the quality of a display. These visual properties pertain to the spatial, temporal and luminance domain. Spatial

Ergonomics Group, University of Twente. PO Box 217, 7500 AE Enschede The Netherlands. Tel: 053-893304/893346. Fax: 053-356695. E-mail: Spenkelink\%erg\%WMW@civp.utwente.nl 
factors are the size and shape of the pixels of a display (which are named display elements or dels); the spatial separation of dels; the physical size of the display and all factors related to the geometry of objects-to-be-displayed, e.g. the font matrix. Temporal aspects are rise and decay times of phosphors or their equivalents. This is the time that a del needs to switch fully from an off-state to an on-state and vice versa. Liquid crystal displays are renowned for problems in this respect. Further temporal aspects are the display refresh rate and the effects of moving displayed objects. Luminance aspects are basically foreground luminance, background luminance (and thereby luminous contrasts), and spectral factors (including colour contrast). All these physical properties have perceptual correlates such as brightness and colour. These factors are of specific interest to us.

This study focuses on two prominent FPD features: the physical separation between dels (the 'gap') and the basic shape of the dels. In addition the height/width ratio of the del is studied in relation to a fixed font matrix.

The dels of a liquid crystal display (LCD) or electroluminescence display (ELD) are activated by the application of an electrical current. In order to be able to activate single dels, they have to be insulated, and this is achieved by separation with an insulating material. The resulting (square) grid adds to the non-active area of the display and in typical FPDs amounts to $20-70 \%$ of the total display area. This quantity is usually expressed as the ratio of active to inactive display area: the fill factor. In combination with a very steep luminance gradient compared to ordinary CRTs, the result is that the dels are visibly separated. Furthermore, the spatial frequency of the display raster is in the order of 10 to 40 cycles/degree visual angle. These frequencies are above the visual threshold at moderate contrasts (Murch and Virgin, 1985). To make things worse, the human visual system is particularly sensitive to the square wave luminance profile of an FPD compared to the sinusoidal profile of a CRT (Benzschawel et al., 1986). The (unwanted) contrast between the gap and either the 'on' dels or the 'off' dels can be decreased by choosing an appropriate colour (and thus luminance) for the gap. Technically this is possible by dyeing the gap material. But what colour is to be preferred? If one chooses a gap in foreground colour, the quality of the display area in the 'on' state (eg. text characters) will improve, but at the cost of introducing a gap-grid in the background. If, on the other hand, one chooses a gap in background colour, the image will consist of visibly separated dels and alphanumerics will be seen to consist of individual dots. Figure 3 demonstrates these differences for square and round dels.

As mentioned before, FPD dels are characterized by a steep luminance gradient compared to CRTs. Due to the steep luminance gradients these dels are perceived as sharply defined. Sharpness here refers to a perceptual quality that is often attributed to selective sensitivity of the visual system to different spatial frequencies (Ginsburg, 1986) and to the perception of local contrasts (Kokoscha, 1986). One negative effect of sharp dels is that adjacent 'on' dels which are not in the principal direction of the raster (i.e. in oblique lines) cause a strong staircase effect (jagged lines). Rounding off the corners of dels may counteract this phenomenon. On the other hand a display with round dels will, given a square raster, have a smaller fill factor. Nevertheless it may still comply with the 
standards (ANSI, 1988; ISO, 1988). The effects of del shape are demonstrated in Figures 2 and 3.

The elongation or height/width ratio of a del has a pronounced effect on the appearance of fixed matrix size fonts. This ratio is co-determined by the dimensions of the del. A broad del produces a broad character matrix and thus broad characters. A slim del produces slim characters. The del dimensions also interact with the gap: a broad del produces widely spaced (vertical) gaps, a slim del gives finely spaced gaps. The height/width ratio of a font also plays a major part in display standards. Due to the intricate relations between a large number of spatial font parameters it is often very difficult, if not impossible, to meet the requirements by manipulation of the font matrix. In practice there are not so many degrees of freedom in the design. The geometrical properties of a display and its dels largely dictate the matrix structure. This makes the mutual dependency between font characteristics and del proportions interesting. Given a certain character matrix, compliance with the height/width and size requirements for alphanumerics may be achieved by proper dimensioning of the dels.

In previous research (Spenkelink et al., 1990b; Spenkelink, 1990) it was shown that the appreciation of font characteristics was independent of the subjective assessment of characteristics in the luminance domain ie. brightness, contrast and colour. Although these characteristics co-vary with the basic shape of dels and type of separation between dels, it is an open question whether these latter characteristics can also be assessed independently of the font and vice versa.

A number of questions emerge. The first question concerns the effect of separation or local luminance variations on perceived display quality and how to improve quality by manipulating the gap colour. A second question asks if round dels may effectively counteract the staircase phenomenon. Further, can the display font be optimized by manipulating the height or width of the dels? Altering the size of dels affects the gap pattern, which becomes finer or coarser. Thus del size may interact with the type of gap between dels and with the shape of the del. Related to this is the question whether subjects can assess the quality of the font independent of other display characteristics that are varied. The last question concerns the relation between subjective measures and objective display characteristics, especially the contrast and del size.

The importance of ergonomic knowledge in display development and design was recognized in the ESPIRT project OS 1593, 'modelling and simulation of modern display technologies under office work conditions'. In this project a display simulator was developed and built for dynamic simulations of FPD's, notably liquid crystal displays and electroluminescence displays with, "the aim ... to identify user requirements for flat panel display developments" (Placencia Porrero et al., 1989). A number of methods for ergonomics research have been implemented on the simulator (Spenkelink et al., 1990b). Several studies have concentrated on the subjective assessment of flat panel display quality, using the rating technique. The rating scale and its development are described in (Spenkelink et al., 1990a). The individual items of the scale pertain to different perceivable aspects of the display (eg. brightness, colour contrast, shape of alphanumerics) in the three domains mentioned before. The current research is carried out on the display simulator and uses some of the concepts 


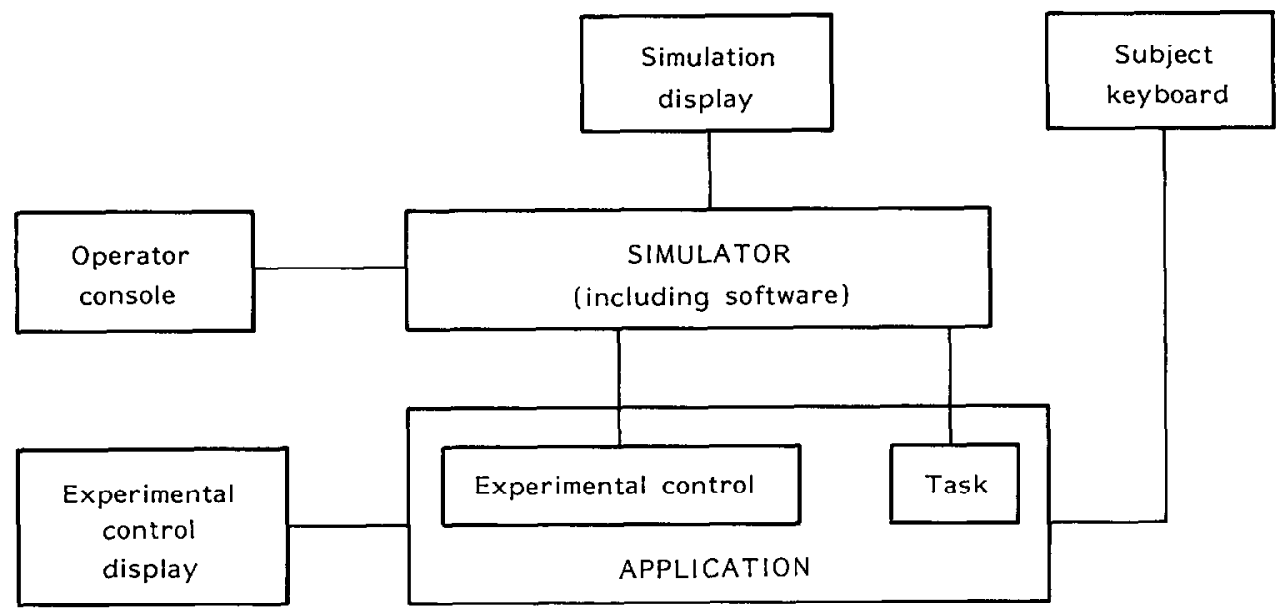

Figure 1. Schematic view of configuration of display simulator

developed with the rating scale, albeit in a somewhat different setting. Instead of ratings, preference data is collected. It is evident that perceived display quality is not unidimensional (eg. Spenkelink et al., 1990b) and therefore multiple preferences pertaining to different characteristics are collected.

\section{The experiment}

\section{The simulator}

The simulator configuration used in the experiment is shown in Figure 1. To create a high degree of flexibility, the task and the experimental procedure have been completely separated. Further the stimulus material (the displayed information) is independent of both the task and the experimental procedure. For technical and performance specifications of the simulator the reader is referred to Placencia Porrero et al. (1989).

In order to define a simulation, FPD parameters in the spatial, luminance and temporal domain have to be specified. The parameters in the spatial domain have to be mapped onto the simulation display with its own spatial properties.

In order to achieve, for example, rectangular simulated display elements, a number of simulation CRT pixels have to be used. The more pixels are used, the finer the detail that can be simulated. The ultimate size of the simulated display elements and their gaps depends on the smallest feature to be simulated, which is in all cases the gap between actual FPD display elements. The larger visual angle that simulated images extend is corrected for by increasing the viewing distance.

The simulated displays and images: stimulus material

Eighteen different images were defined, varying in three respects:

- the size of the display elements, 
Table 1. Independent variables in the pairwise comparison study

\begin{tabular}{lll}
\hline $\begin{array}{l}\text { Independent } \\
\text { variable }\end{array}$ & \multicolumn{1}{c}{ Value } & \multicolumn{1}{c}{$\begin{array}{c}\text { Example } \\
\text { (in Figures) }\end{array}$} \\
\hline Del size & $5 \times 5$ (simulation CRT & \\
& $4 \times 6$ pixels; $\mathrm{H} \times \mathrm{V}$ ) & Figure 2(a) \\
Del shape & $4 \times 7$ & Figure 2(b) \\
& Square & Figures 2(a), right; 2(b), left \\
Type of gap & Rounded & 2(a), left; 2(b), right \\
& $\begin{array}{l}\text { No gap } \\
\text { Gap in foreground colour }\end{array}$ & Figure 2(a) \\
& Gap in background colour & Figure 2(b) \\
\hline
\end{tabular}

- the shape of the display elements,

- the type of separation between display elements.

The 'no gap' condition is representative of displays with a gap that is smaller than the visual system can detect. Table 1 lists the independent variables and their respective values.

Figures 2(a) and 2(b) show simulated displays used in the experiment with a gap in foreground colour and in background colour respectively.

The type of gap strongly influences the appearance of the display. In the display shown in Figure 2(a) the gap 'fills' the characters, but introduces a grid in the background and decreases the mean luminance of the background and thereby the global contrast between foreground and background. In the display of Figure 2(b) the background is homogeneous with respect to luminance/ colour, but the characters are non-contiguous: the dels individually stand out against the background. It may be assumed that the perception of display quality is co-dependent on the 'type of gap' factor.

Figure 3 depicts all combinations of del shape, del size and type of gap. Each sub-figure shows an area of $4 \times 4$ simulated dels. On-dels are in black, the background area is white. The displayed pattern consists of eight on-dels. This

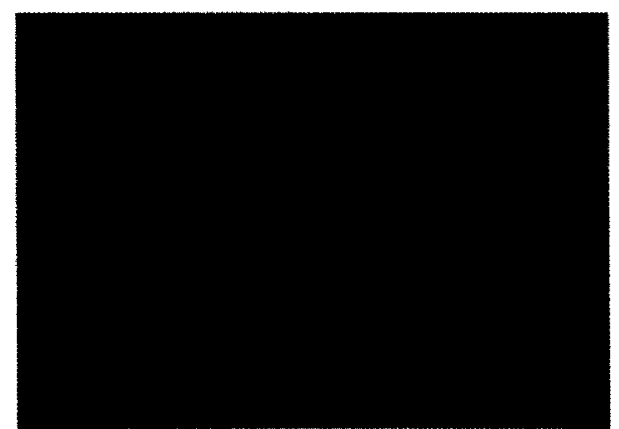

a

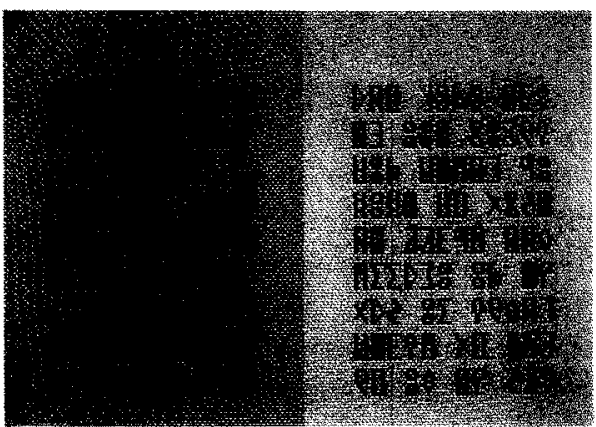

b

Figure 2. Two examples of half-screen images used in paired comparison task. See also Table 1 
Square del
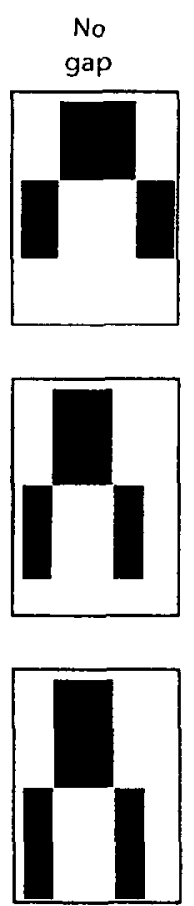
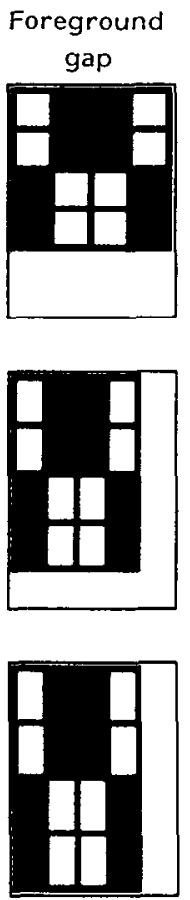
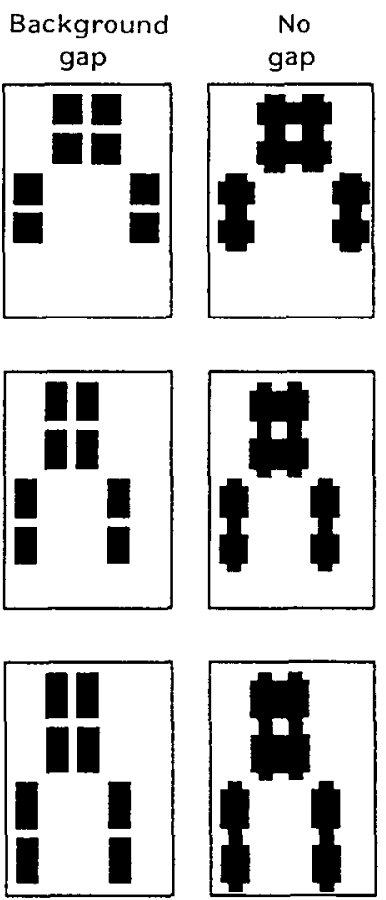

Round del

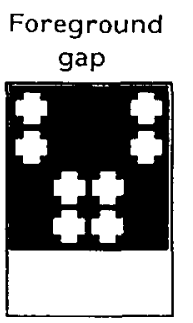

Background

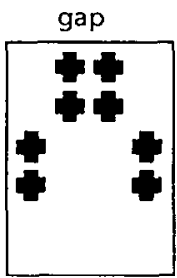

$5 \star 5$ del
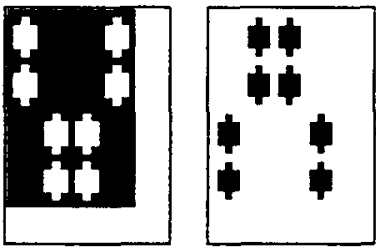

$4 * 6$ del

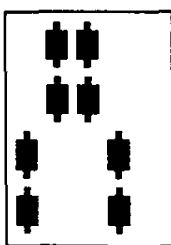

Figure 3. Schematic drawing showing the effects of gap colour, del shape and del size for a black on white display

can best be seen in the sub-figures with a background gap. The scale of Figure 3 is approximately $4: 1$. In order for the depicted dels to extend the same visual angle as the images in the experiment, Figure 3 should be viewed from $6 \mathrm{~m}$.

On most FPDs the dels are square (rectangular). It is conceivable however, that other del shapes can be defined. In this study both square and round dels were defined. The round dels were identical in size and height/width ratio to the square ones, except that the corners were 'rounded' by defining a separate colour for the corner pixels of the simulated dels. When no gap was present, the corner pixels of the round dels were defined in background colour (producing little white spots in the character area). When a gap was present the colour of the corner pixels was the same as that of the gap.

The experimental, simulated images consisted of black 'nonsense'-characters on a white ground. Each image was of size $210 \times 148.5 \mathrm{~mm}$. The characters were designed to incorporate the relevant characteristics of normal alphanumerics (horizontal lines, vertical and slanted lines, curvatures etc.) without creating a strong impression of actually being alphanumerics. This was done to circumvent the problem that subjects might have strong convictions about what characters ought to look like. The characters used in the present study are shown in Figure 2. Although the characters were identical in each image as far as the dot matrix is concerned, the appearance of the characters differed because of the variations in geometrical properties. Table 2 shows the most important 
Table 2. Del size and font characteristics

\begin{tabular}{lcccc}
\hline & $\begin{array}{c}\text { Character } \\
\text { height (CH) }\end{array}$ & $\begin{array}{c}\text { Height/width } \\
\text { ratio }\end{array}$ & $\begin{array}{c}\text { Stroke width } \\
(\mathrm{SW})\end{array}$ & $\begin{array}{c}\text { Character } \\
\text { spacing (CS) }\end{array}$ \\
\hline Unit & Minute of arc & Ratio & $\mathrm{CH}$ & SW/CH \\
\hline $5 \times 5$ del & $\mathbf{1 7 . 3}$ & $\mathbf{0 . 7 8}$ & 0.22 & $\mathbf{1 / 0 . 2 2}$ \\
$4 \times 6$ del & 26.7 & $\mathbf{0 . 5 2}$ & $\mathbf{0 . 1 5}$ & $1 / 0.15$ \\
$4 \times 7$ del & 31.2 & 0.44 & $\mathbf{0 . 1 3}$ & $\mathbf{1} / 0.13$ \\
\hline Norm & $16-22$ & $0.5-0.9$ & $0.12-0.17$ & $1 / 0.2-0.5$ \\
\hline
\end{tabular}

font parameters for the del sizes used in the experiment. The entries in the table may be compared to the requirements from a number of display standards (ANSI, 1988; ISO, 1988; Ericsson Information Systems, 1983). These requirements are shown as a range of values in the last row of the table. Bold entries are within the range of required values.

Finally, Table 3 lists important simulation and environmental parameters that were kept constant throughout the study.

\section{Method}

Paired comparisons were used to collect subjective preferences. In the paired comparison paradigm the experimental subjects are confronted with two stimuli (conditions) at a time and are required to make some sort of comparison between the two stimuli e.g. to indicate which of the two stimuli is preferred. In a typical paired comparison procedure, all possible pairs of stimuli are evaluated by the subjects. The resulting two-way table of preferences (or whatever other measure is used) can be analysed in a number of ways. A straightforward analysis involves ranking the stimuli according to the number of times they are preferred.

The paired comparison method was chosen mainly for its efficiency. Many different stimuli can be processed in a short time, even in a complete factorial design. It is most efficient if a subject evaluates each pair only once. Replication

Table 3. Important parameters in the pairwise comparison study

\begin{tabular}{lcc}
\hline Parameter & Value & $\begin{array}{c}\text { Unit of } \\
\text { measurement }\end{array}$ \\
\hline Foreground luminance & 1.5 & $\mathrm{~cd} / \mathrm{m}^{2}$ \\
Foreground colour & $0.333,0.333$ & $x, y$ \\
Background luminance & 11.5 & $\mathrm{~cd} / \mathrm{m}^{2}$ \\
Background colour & $0.333,0.333$ & $x, y$ \\
CRT dels (H \& V) & 0.232 & $\mathrm{~mm}$ \\
Viewing distance & 161 & $\mathrm{~cm}$ \\
Ambient illumination & 300 & $\mathrm{Lux}$ \\
(horizontal at 72cm height) & & \\
\hline
\end{tabular}


Table 4. Inter-rater reliability coefficients

\begin{tabular}{lcc}
\hline Item & $r_{k k}$ & Critical $r_{k k}$ \\
\hline Sharpness & 0.969 & 0.412 \\
Contrast & 0.928 & 0.412 \\
Character & 0.906 & 0.412 \\
Overall & 0.938 & 0.412 \\
\hline
\end{tabular}

can be achieved by using several raters. In order to justify such a replication procedure, the inter-rater reliability should be high.

A further question of interest concerns the applicability of the concepts from our rating procedure in a paired comparison paradigm and the suitability of the multidimensional scaling techniques we apply in the analysis of such rating data for analysis of the comparison data.

Since we are interested in display characteristics (which can be assessed objectively), there is the need to establish the relation between those characteristics and the data obtained through paired comparison. A number of display characteristics were measured.

\section{Task and procedure}

First the subjects received written instructions concerning their task and the experimental procedure. Then they were asked to study a written description of the aspects that had to be used in evaluating the stimulus pairs.

The 18 different experimental stimuli were presented to the subjects in 153 $(0.5 n \times n-1)$ pairs in a random order at a viewing distance of $161 \mathrm{~cm}$. One of the images in a pair occupied the left half of the simulation display and the other the right half. For each of the pairs the subjects separately indicated their preference for one of the two images with respect to the sharpness, the contrast and the appearance of the characters (in this order). Finally they indicated their 'overall' preference for one image of the pair.

Sixteen subjects voluntarily participated in the study. All were students of the University of Twente. None of them had previous experience of being a subject in an experiment. All subjects had normal or corrected-to-normal vision.

\section{Results}

The coefficient of consistence (Kendall, 1962) was calculated for each subject and item. The mean coefficient for the sharpness, contrast, font and overall comparisons were $0.790,0.726,0.726$ and 0.786 respectively. It may be concluded from these figures that the subjects were at least reasonably consistent. The chi-square test proposed by Kendall gives a probability of over $99.9 \%$ that the number of inconsistencies is larger when gambling is involved.

The intra-class correlation (Winer, 1972) was calculated for each of the four preference items. This statistic may be interpreted as a measure of inter rater reliability (see Table 4). Coefficients higher than 'critical $r_{k k}$ ' are significant correlations $(p<0.05)$. Note that all coefficients are well above the critical value.

The preference data of the preference items (excluding the overall item) were 
analysed by individual differences scaling (INDSCAL) (as implemented in the mainframe version of the Statistical Package for the Social Sciences). INDSCAL is a multidimensional scaling technique which preserves differences in views between the subjects. Multidimensional scaling techniques

"... use proximities among any kind of objects as input. A proximity is a number which indicates how similar or how different two objects are, or are perceived to be, or any measure of this kind. The output is a spatial representation, consisting of a geometric configuration of points, as on a map. Each point in the configuration corresponds to one of the objects. This configuration reflects the 'hidden structure' in the data, and often makes the data much easier to comprehend. By reflecting the data structure we mean that the larger the dissimilarity between the two objects, as shown by their proximity value, the further apart they should be in the spatial map" (Kruskal and Wish, 1978).

Input to the INDSCAL routine consisted of the number of times that individual images were preferred. A good INDSCAL solution for the present data was found in two dimensions, with a squared correlation (RSQ) or percent of variance accounted for of 0.816 . RSQ is considered a good indicator for the appropriateness of the solution (Kruskal and Wish 1978; Schiffman et al., 1981). The solution is shown in Figure 4 . The units on both axes of Figure 4 are arbitrary.

It can be seen in Figure 4 that the displays with different del sizes are drawn apart in the vertical direction, whereas the del shape and type of gap factor are strongly present in the horizontal direction. Further, the subjects mainly spread in the vertical direction.

We applied a vector interpretation to the INDSCAL solution and used multiple regression to fit the below mentioned measures to the configuration. From measurement of mean foreground- and mean background-luminance of the simulation display two contrast indices were calculated:

$$
\begin{aligned}
& K=\mathrm{L}_{\max } / \mathrm{L}_{\min } \\
& \mathrm{L}_{\bmod }=\left(\mathrm{L}_{\max }-\mathrm{L}_{\min }\right) /\left(\mathrm{L}_{\max }+\mathrm{L}_{\min }\right)
\end{aligned}
$$

where $K$ is contrast ratio and $\mathrm{L}_{\bmod }$ is luminance modulation, $\mathrm{L}_{\max }$ is the area with highest luminance (in the present case the background) and $L_{m i n}$ is the area with lowest luminance (the foreground). Regression of $\mathrm{L}_{\text {mod }}$ on the INDSCAL solution is highly significant in the horizontal dimension $(p<0.0001$; RSQ 0.974) and has a significantly higher RSQ than the contrast ratio (RSQ 0.845 ). Therefore $\mathrm{L}_{\text {mod }}$ is further used in the data analyses.

Of the individual items, the sharpness preference contributes most to the variance in the first dimension ( $p<0.0001$, RSQ 0.0933). In addition, regression of $\mathrm{L}_{\text {mod }}$ on sharpness is highly significant $(p<0.0001, \mathrm{RSQ} 0.939$ ).

The ratio of height/width of a del shows a significant regression on the vertical dimension ( $p<0.0001$, RSQ 0.893).

The overall preference produces a close fit with the INDSCAL solution with $p<0.0001$, RSQ 0.768. This is mainly due to the first dimension $(p<0.0001, \mathrm{RSQ}$ 0.726 ). Further, the overall preference is a weighted linear combination of the 


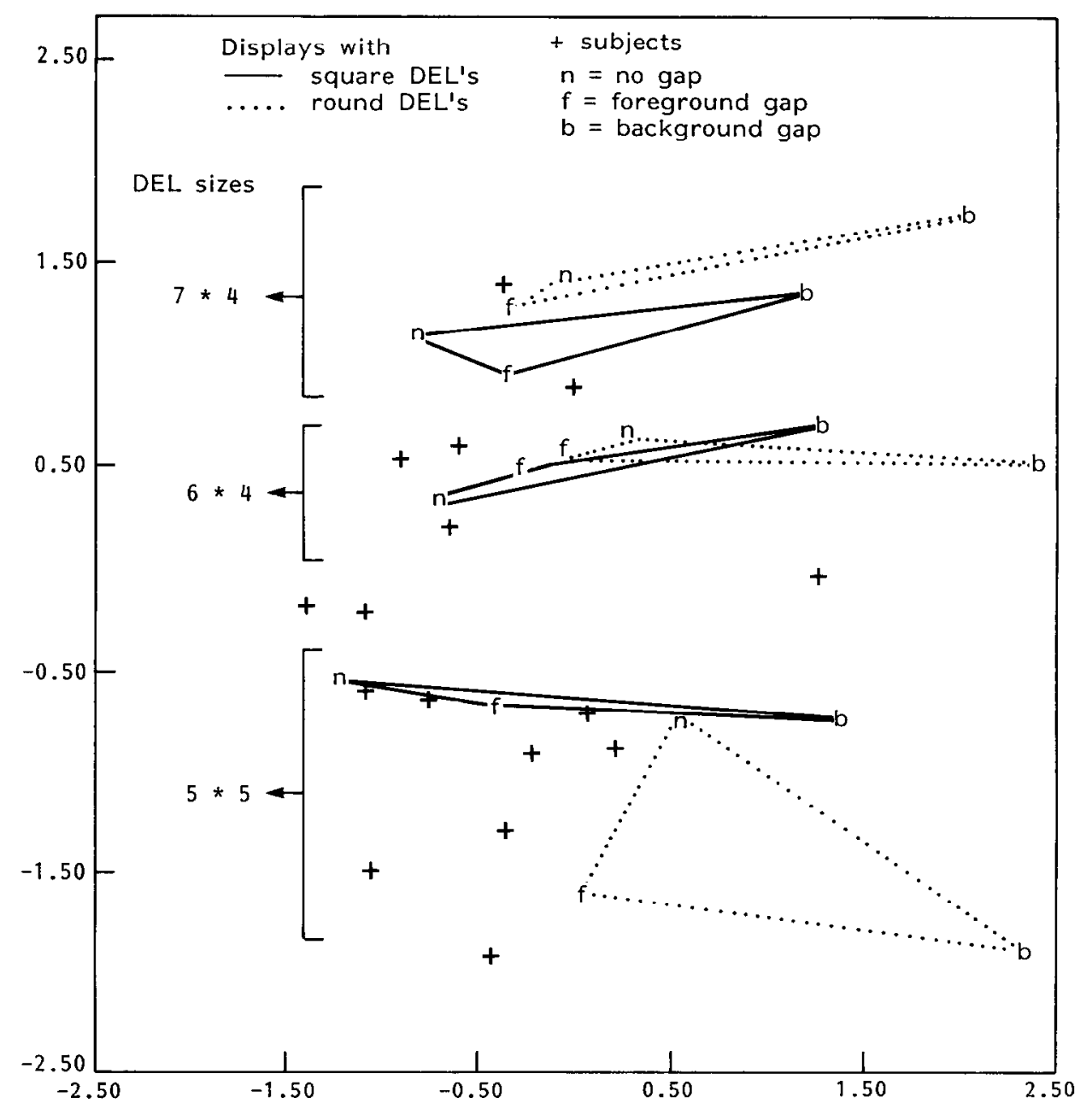

Figure 4. 2D INDSCAL solution for stimuli and subjects. Solution based on simultaneous assessment of preferences for sharpness, contrast and character

three other items: $p<0.0001$; RSQ 0.988 ; beta weights 0.565 (contrast), 0.391 (character) and 0.132 (sliarpness).

The preference data were tested for normality with the Kolmogorov-Smirnov test. After a positive result, the data pertaining to each of the items were subjected to analysis of variance with 3 (del size) $\times 2$ (del shape) $\times 3$ (type of gap) $=18$ conditions. The results are presented in Table 5. Significant main effects in the ANOVA were tested separately with $t$-tests for differences between pairs. The result is given in the rightmost column of the table (' $>$ ' means 'significantly preferred over').

\section{Discussion}

The coefficients of consistence are reasonably high. Nevertheless inconsistencies are present in the data. 
Table 5. ANOVA and $t$-tests on preference data for the four items. Significant effects ( $p<0.05$; two-sided for $t$-tests) only. $t$-tests only for main effects

\begin{tabular}{|c|c|c|c|c|c|c|c|}
\hline \multirow[t]{2}{*}{ Item } & \multicolumn{5}{|c|}{ ANOVA } & \multirow{2}{*}{\multicolumn{2}{|c|}{$\begin{array}{c}t \text {-tests } \\
\text { Significant effects }\end{array}$}} \\
\hline & Effect & SS & DF & $\mathrm{F}$ & $\mathrm{p}$ & & \\
\hline \multirow[t]{2}{*}{ Sharpness } & DELSHAPE & 953.389 & 1 & 131.317 & 0.000 & \multirow{2}{*}{$\begin{array}{l}\text { square } \\
\text { none } \\
\text { fore }\end{array}$} & $>$ round \\
\hline & GAPTYPE & 3141.396 & 2 & 216.344 & 0.000 & & $\begin{array}{l}>\text { back } \\
>\text { back }\end{array}$ \\
\hline \multirow[t]{3}{*}{ Contrast } & DELSHAPE & 566.722 & 1 & 52.019 & 0.000 & \multirow{2}{*}{\multicolumn{2}{|c|}{$\begin{array}{l}\text { square }>\text { round } \\
\text { none }>\text { fore } \\
\text { none }>\text { back } \\
\text { fore }>\text { back }\end{array}$}} \\
\hline & GAPTYPE & 1846.688 & 2 & 84.754 & 0.000 & & \\
\hline & DELSHAPE $\times$ GAPTYPE & 241.840 & 2 & 11.099 & 0.000 & \multirow{4}{*}{$\begin{array}{l}5 \times 5 \\
4 \times 6 \\
\text { square } \\
\text { none } \\
\text { fore }\end{array}$} & \\
\hline \multirow[t]{3}{*}{ Character } & DELSIZE & 1165.646 & 2 & 47.934 & 0.000 & & $\begin{array}{l}>4 \times 7 \\
>4 \times 7\end{array}$ \\
\hline & DELSHAPE & 276.125 & 1 & 22.569 & 0.000 & & $>$ round \\
\hline & GAPTYPE & 878.813 & 2 & 35.915 & 0.000 & & $\begin{array}{l}>\text { back } \\
>\text { back }\end{array}$ \\
\hline \multirow[t]{5}{*}{ Overall } & DELSIZE & 240.896 & 2 & 11.026 & 0.000 & \multirow{5}{*}{$\begin{array}{l}5 \times 5 \\
4 \times 6 \\
\text { square } \\
\text { none } \\
\text { fore }\end{array}$} & $>4 \times 7$ \\
\hline & & & & & & & $\begin{array}{l}>4 \times 7 \\
>\text { round }\end{array}$ \\
\hline & GAPTYPE & 1992.896 & $\begin{array}{l}1 \\
2\end{array}$ & 91.216 & 0.000 & & $>$ back \\
\hline & & & & & & & $>$ back \\
\hline & DELSHAPE $\times$ GAPTYPE & 119.965 & 2 & 5.491 & 0.005 & & \\
\hline
\end{tabular}

* due to round dels (see also Figure 6).

The results of the reliability analysis as shown in Table 4 indicate that inter-rater reliability of the preferences is high. This means that a high degree of preference agreement existed between the raters. Since this argument is valid for all four preference items it seems valid to state also that the raters had similar conceptions of the items of contrast, sharpness and character shape and consequently agreed with respect to the overall preference.

The regression of the items of sharpness, contrast and character shape on the overall preference indicate that the three separate items linearly combined into an overall preference. Further support for this comes from the ANOVA: all significant main effects for the separate items are retained in the overall preference.

The INDSCAL analysis produced a usable solution. The RSQ of 0.816 indicates quite a good model fit. The geometrical representation discriminates between the independent variables and the levels thereof. Further, there is a good correspondence between configuration and the results of the ANOVA.

The physical parameters of delsize and luminance modulation give a good fit with the subjective data. The horizontal dimension of the INDSCAL solution corresponds almost perfectly with the luminance modulation, whereas the vertical dimension shows a good correspondence with the del height/width ratio.

The preference for images differed with the type of gap, i.e. images with a 


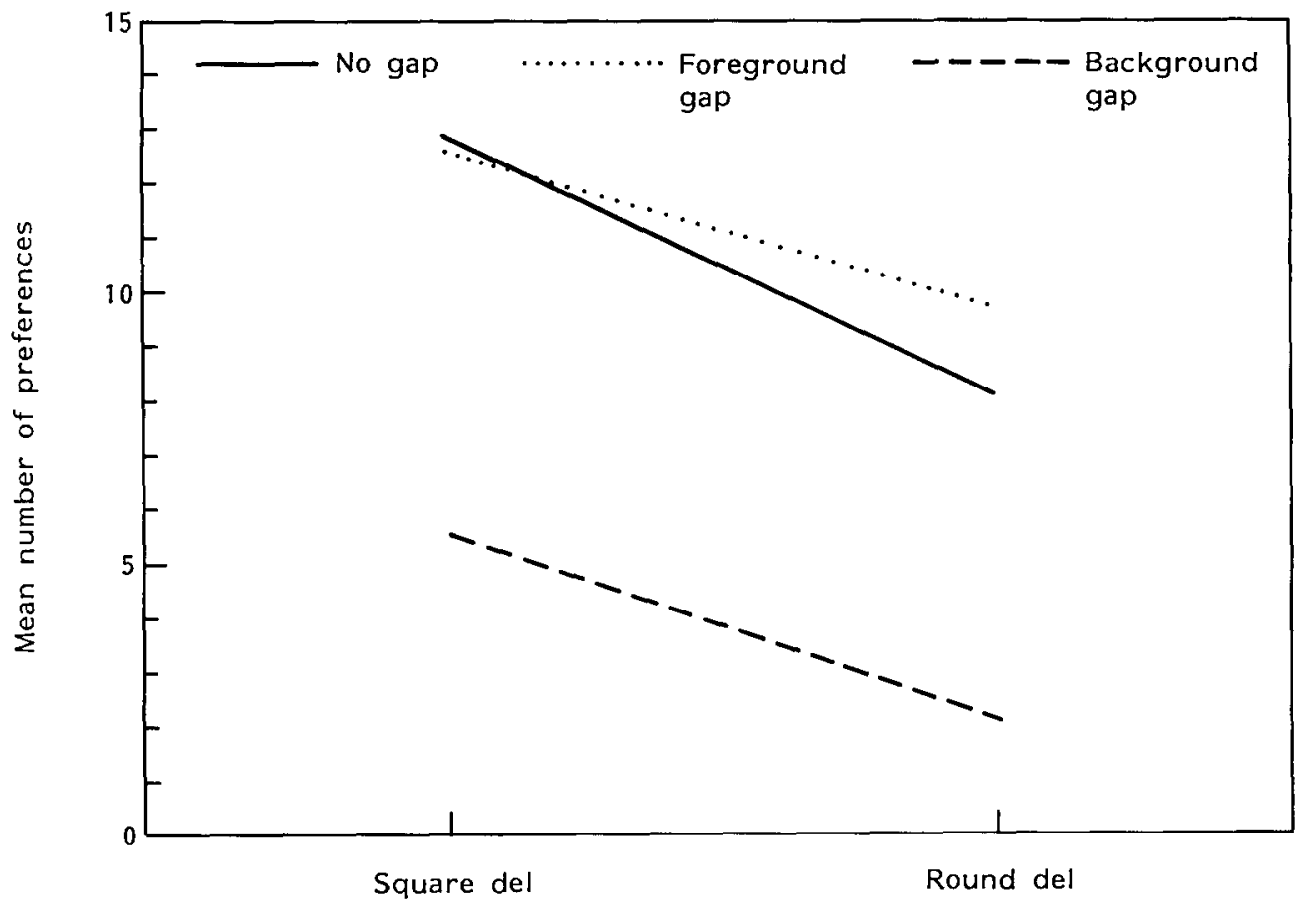

Figure 5. Interaction between del shape and type of gap. Data based on item of sharpness

background gap were significantly less preferred than both images without gap and images with a foreground gap. With respect to the contrast preference there was also a significant higher preference for images without gap compared to images with a foreground gap. An interpretation of these results is that the integrity of the foreground is more important than that of the background.

In the INDSCAL solution the differences in preference related to the type of gap can also be distinguished (in the first dimension). For square dels images without gap are preferred (see first INDSCAL dimension) over those with a foreground gap. With round dels, on the other hand, the foreground gap images score a little more positively than the images without gap. This inversion of preference may seem strange, but corresponds with the measured luminance modulation which, contrary to images with square dels, for images with round dels is better when a foreground gap was present. The reason for this is that the corner pixels of the round dels were defined in background colour if a gap was absent, but in foreground colour if a foreground gap was present. Again the integrity of the foreground appears to be very important. Figure 5, depicting the preference data for the sharpness item, shows this effect.

With respect to the shape of the dels, the INDSCAL solution indicates a general advantage for square dels compared to round dels. This is confirmed by the ANOVA, but there is also an interaction with the type of gap for contrast preference. A glance at this interaction between the shape of the dels and the 


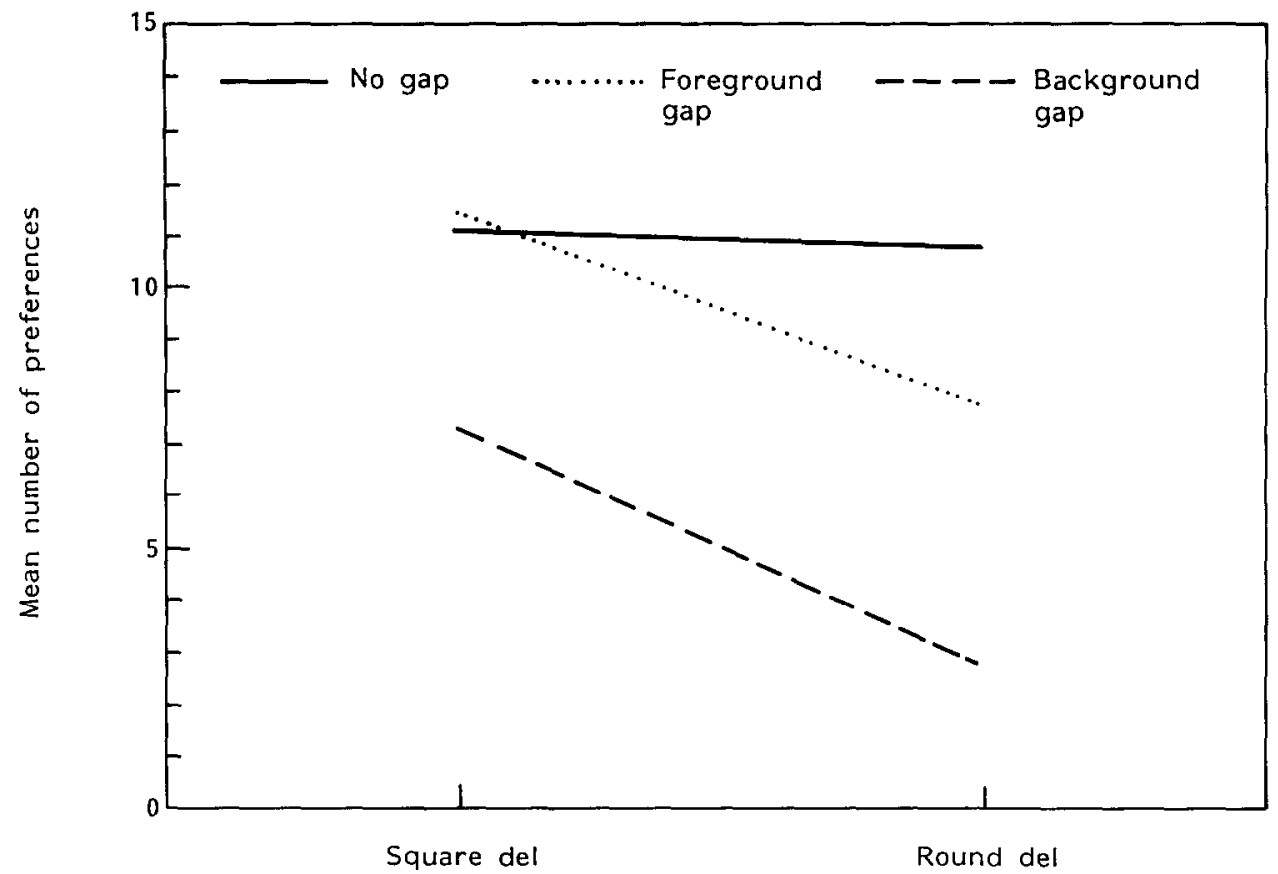

Figure 6. Interaction between del shape and type of gap. Data based on item of contrast

type of gap (see Figure 6) makes clear that images with round dels are at a disadvantage whenever a gap is present.

The images with different del sizes are vertically drawn far apart in Figure 4. The ANOVA results indicate the del size effect to be significant. The complementary $t$-tests indicate that the $4 \times 7$ del is at a disadvantage compared to the $5 \times 5$ and the $4 \times 6$ del. Regression shows that the ratio of height/width of the del is highly significant in the second dimension of the INDSCAL solution. The disadvantage of the $4 \times 7$ del over the other two dels can also be inferred from Table 2, were the font in the $4 \times 7$ del images conforms to the standards only with respect to the stroke width. Finally, no interaction was found between del size and type of gap, which interaction might have been interpreted as a gap-grid size effect.

\section{Conclusions}

This study examined subjective considerations of flat panel display quality. The data from the paired comparisons exhibit a sufficiently high inter-rater reliability to allow further analyses.

The display characteristics of del shape and del size appeared to be important factors in the preference judgements of the subjects. This was also true for the type of gap.

The luminance modulation, which varied between experimental images because of relative differences in the display area of foreground colour and 
background colour respectively, was fitted almost perfectly in the first dimension of the multidimensional scaling solution. In this dimension we also located the type of gap factor and the del shape factor. These two factors were actually responsible for the differences in the luminance modulation. Therefore the linear relation between the luminance modulation and the sharpness preferences and between both these measures and the first dimension of the scaling solution should be understood in this light. It is possible, and the sharpness preference data does suggest it, that subjects did not react directly to the type of gap or the del shape, but to the resulting differences in luminance modulation.

The solution for the 'gap-problem' is to design displays with gaps in foreground colour, which is in fact optimizing the integrity of the foreground or character area by avoiding visible gaps in this area.

With respect to the font, it may be concluded that the subjects were able to assess the appropriateness of character shape independently of other characteristics that were varied in the experiment. Furthermore, both the $5 \times 5$ and $4 \times 6$ dels produced the most acceptable characters. This is in line with the standards for the height/width ratio of alphanumerics. It also shows that it may be worthwhile to design dels and font interactively instead of designing a font for a given display. Such a procedure increases the opportunities to conform to the standards.

\section{References}

American National Standards Institute, (1988) American National Standard for Human Factors Engineering of Visual Display Terminal Workstations ANSI

Benzchawel, T., Rogowitz, B.E. and Rappoport, E. (1986) 'Detection of undersampled gratings', SID International Symposium, Digest of Technical Papers New York, USA, $432-435$

Ericsson Information System (1983) Ergonomic Principles in Office Automation Ericsson Information Systems AB, Bromma, Sweden

Ginsburg, A.P. (1986) 'Spatial filtering and visual form perception' in Boff, K.R. et al. (eds) Handbook of Perception and Human Performance Wiley, chapter 34

International Standards Organization (1988) Visual Display Terminals Used for Office Tasks - Ergonomic Requirements, Part 3: Visual Display Requirements, Draft International Stardard ISO/DIS 9241-3 ISO

Kendall, M.G. (1982) Rank Correlation Methods Charles Griffin \& Co

Kokoschka, S. (1986) 'Visibility aspects of VDUs in terms of contrast and luminance' Behaviour and Inf. Technol. 5, 4, 309-333

Kruskal, J.B. and Wish, M. (1978) Multidimensional Scaling Sage Publications, Beverley Hills, CA, USA

Murch, G. and Virgin, L. (1985) 'Resolution and addressability: how much is enough?' SID International Symposium, Digest of Technical Papers Palisades Institute for Research Services Inc., New York, USA, 101-103

Placencia Porrero, I., Levis, J.P., Duprez, J., Bosman D. and Spenkelink, G. (1989) Modelling and simulation of the visual characteristics of flat panel display technologies under office work conditions' Esprit' 89 Conf. Proc. Brussels, 915-933

Schiffman, S., Reynolds, M., Young, F. (1981) Introduction to Multidimensional Scaling Academic Press 
Spenkelink, G. (1990) 'A validation study of the High End Simulator' Internal Report for the EC in the Project Espirt OS 1593

Spenkelink, G., Besuijen, K. and White, T. (1990a) 'Evaluation of flat panel display properties on a high fidelity simulator' Proceedings of Interacting With Computers: Preparing for the Nineties MCI working group of the Dutch Computer Society, Noordwijk

Spenkelink, G., Spijker, H. van and White, T. (1990b) 'Evaluation of flat panel display properties on a high fidelity display simulator' in Diaper, D. et al. (eds) Interact '90, Proc. IFIP TC 13 3rd Conf. Human-Computer Interaction Cambridge, UK, 721-724

Winer, B.J. (1972) Statistical Principles in Experimental Design McGraw Hill 